\title{
Stochastic differential equations with random coefficients
}

\author{
ARTURO KOHATSU-HIGA ${ }^{1 *}$, JORGE A. LEÓN ${ }^{2}$ and DAVID NUALART ${ }^{3}$ \\ ${ }^{1}$ Universitat Pompeu Fabra, Departament d'Economia, Ramon Trias Fargas 25-27, 08005 \\ Barcelona, Spain \\ ${ }^{2}$ CINVESTAV and CIMAT, Departamento de Matemáticas, Apartado Postal 14-740, 07000 \\ México D. F., Mexico \\ ${ }^{3}$ Facultat de Matemàtiques, Universitat de Barcelona, Gran Via 585, 08007 Barcelona, Spain
}

In this paper we establish the existence and uniqueness of a solution for different types of stochastic differential equation with random initial conditions and random coefficients. The stochastic integral is interpreted as a generalized Stratonovich integral, and the techniques used to derive these results are mainly based on the path properties of the Brownian motion, and the definition of the Stratonovich integral.

Keywords: stochastic differential equations; Stratonovich integrals

\section{Introduction}

Suppose that $W=\left\{W_{t}, t \in[0,1]\right\}$ is a standard Wiener process. The trajectories of $W$ do not have bounded variation, and stochastic integrals such as $\int_{0}^{1} \phi_{s}(\omega) \mathrm{d} W_{s}(\omega)$ cannot be defined pathwise. A natural approach to define stochastic integrals of non-adapted processes (Nualart and Pardoux 1988) is to take the limit in probability of $\int_{0}^{1} \phi_{s} \dot{W}_{s}^{n} \mathrm{~d} s$, where $W^{n}=\left\{W_{t}^{n}, t \in[0,1]\right\}$ is a sequence of polygonal approximations of $W$. This definition generalizes the Stratonovich integral in the case of adapted processes, but it is not easy to obtain $L^{p}$ estimates for this type of integral unless the integrand is a continuous semimartingale or it has bounded variation paths.

Using the techniques of the Malliavin calculus, one can decompose the Stratonovich integral as the sum of the Skorohod integral (the adjoint of the derivative operator) plus a complementary term expressed in terms of the trace of the derivative operator. Furthermore it is possible to derive a change-of-variables formula for the Skorohod and Stratonovich integrals (Nualart and Pardoux 1988; Russo and Vallois 1993).

The Stratonovich integral follows the rules of the ordinary calculus. This property can be used to formulate and solve stochastic differential equations in the Stratonovich sense. In the case of a random initial condition and constant coefficients, a solution of the form

\footnotetext{
*To whom correspondence should be addressed. e-mail: kohatsu@upf.es
} 
$X_{t}=\varphi_{t}\left(X_{0}\right)$ can be obtained as the composition of the stochastic flow $\varphi_{t}(x)$ associated with the coefficients, with the initial condition $X_{0}$ (Millet et al. 1992). In order to show the uniqueness of this solution, and to handle the case of a random drift, Ocone and Pardoux (1989) have established a generalized Itô-Ventzell formula, using the techniques of the Malliavin calculus. In the one-dimensional case this approach can be applied in connection with Doss representation of the solution (Kohatsu-Higa and León 1997).

In this paper, by means of a direct approach, we construct a solution for the following types of stochastic differential equation with random coefficients, and we show the uniqueness of this solution in the class of processes that are limits of the corresponding approximated equations.

(i) In Section 2 we discuss one-dimensional stochastic differential equations with random initial conditions and random coefficients using the approach introduced by Doss (1977).

(ii) Section 3 is devoted to the particular case where the diffusion coefficient is linear but depends on the time variable.

(iii) Finally in Section 4 we consider two examples of anticipating multidimensional Stratonovich stochastic differential equations that can be treated with our direct approach. First we consider the case of non-random diffusion coefficient, random drift and random initial condition. These equations have been treated by Ocone and Pardoux (1989) by the technique of the stochastic calculus of variations. Secondly, we discuss the equations with boundary conditions studied by Garnier (1995).

In the one-dimensional case (Sections 2 and 3) we shall suppose that the stochastic differential equation is driven by a continuous local martingale. The proof of the main results are based on the classical stochastic calculus and the definition of the Stratonovich integral.

\section{One-dimensional equations in the Stratonovich sense}

Let $Z=\left\{Z_{t}, t \in[0,1]\right\}$ be a continuous semimartingale defined on a filtered probability space $\left(\Omega, \mathrm{F}, P,\left\{\mathrm{~F}_{t}, t, \in[0,1]\right\}\right)$ satisfying the usual conditions. Suppose that $Z$ has a canonical decomposition of the form

$$
Z_{t}=M_{t}+A_{t},
$$

where $M=\left\{M_{t}, t \in[0,1]\right\}$ is a continuous local martingale such that $M_{0}=0$ and $\langle M\rangle_{t}=\int_{0}^{t} m_{s} \mathrm{~d} s$, and $A=\left\{A_{t}, t \in[0,1]\right\}$ is a continuous and bounded variation process.

Given a partition $\pi=\left\{0=t_{0}<t_{1}<\ldots<t_{n}=1\right\}$ we introduce the polygonal approximation of the martingale $M$ associated with $\pi$ by

$$
M_{t}^{\pi}=\int_{0}^{t} \sum_{i=0}^{n-1} \frac{M_{t_{i+1}}-M_{t_{i}}}{t_{i+1}-t_{i}} \mathbf{1}_{\left(t_{i}, t_{i+1}\right]}(s) \mathrm{d} s .
$$

We shall make use of the following notion of stochastic integration with respect to $M$. 
Definition 2.1. Let $Y=\left\{Y_{t}, t \in[0,1]\right\}$ be a measurable stochastic process such that $\int_{0}^{1}\left|Y_{s}\right| \mathrm{d} s<\infty$ a.s. The Stratonovich integral of $Y$ with respect to $M$, denoted by $\int_{0}^{t} Y_{s} \circ \mathrm{d} M_{s}$, is the continuous stochastic process defined by

$$
\lim _{|\pi| \downarrow 0} P\left(\sup _{0 \leqslant t \leqslant 1}\left|\int_{0}^{t} Y_{s} \circ \mathrm{d} M_{s}-\int_{0}^{t} Y_{s} \dot{M}_{s}^{\pi} \mathrm{d} s\right|>\epsilon\right)=0,
$$

for all $\epsilon>0$, where $|\pi|=\max _{0 \leqslant i \leqslant n-1}\left(t_{i+1}-t_{i}\right)$, and $\dot{M}_{s}^{\pi}$ stands for $\mathrm{d} M_{s}^{\pi} / \mathrm{d} s$, provided that this limit exists.

Consider the following stochastic differential equation:

$$
X_{t}=X_{0}+\int_{0}^{t} b\left(X_{s}\right) \mathrm{d} A_{s}+\int_{0}^{t} \sigma\left(X_{s}\right) \circ \mathrm{d} M_{s},
$$

where $X_{0}$ is a given random variable (we do not assume it to be $\mathrm{F}_{0}$ measurable). The coefficients $b, \sigma: \Omega \times \mathbb{R} \rightarrow \mathbb{R}$ are measurable functions verifying the following properties.

(i) For all $\omega \in \Omega$ and $x, y \in \mathbb{R}$ we have

$$
|b(\omega, x)-b(\omega, y)| \leqslant K(\omega)|x-y|,
$$

for some random variable $K$.

(ii) For all $\omega \in \Omega$ the mapping $x \mapsto \sigma(\omega, x)$ is of class $C_{b}^{2}$ (i.e., it has bounded and continuous derivatives of first and second order).

By a solution of (2.1) we mean a continuous stochastic process $X=\left\{X_{t}, t \in[0,1]\right\}$ such that $\left\{\sigma\left(X_{t}\right), t \in[0,1]\right\}$ is Stratonovich integrable with respect to $M$ in the sense of Definition 2.1, and (2.1) holds for all $t \in[0,1]$. Under the above assumptions we can prove the following result.

Theorem 2.2. Let $X_{0}$ be a random variable and $b, \sigma: \Omega \times \mathbb{R} \rightarrow \mathbb{R}$ measurable functions verifying hypotheses (i) and (ii). Then there exists a solution $X$ to (2.1).

Proof. We shall make use of the representation of the solution in the adapted case given by Doss (1977). In order to simplify the notation we shall omit the dependence of the coefficients on $\omega$. Let $h(x, y)$ be the solution of

$$
\begin{aligned}
\frac{\partial h}{\partial y}(x, y) & =\sigma(h(x, y)) \\
h(x, 0) & =x .
\end{aligned}
$$

Define $f(x, y)=b(h(x, y)) \exp \left(-\int_{0}^{y} \sigma^{\prime}(h(x, z)) \mathrm{d} z\right)$. Note that

$$
\frac{\partial h}{\partial x}(x, y)=\exp \left(\int_{0}^{y} \sigma^{\prime}(h(x, z)) \mathrm{d} z\right) .
$$


Let $Y=\left\{Y_{t}, t \in[0,1]\right\}$ be the unique solution of

$$
Y_{t}=X_{0}+\int_{0}^{t} f\left(Y_{s}, M_{s}\right) \mathrm{d} A_{s} .
$$

Define $X_{t}=h\left(Y_{t}, M_{t}\right)$ and $\mathrm{X}_{t}^{\pi}=h\left(Y_{t}, M_{t}^{\pi}\right)$ for any partition $\pi=\left\{0=t_{0}<t_{1}<\ldots\right.$ $\left.<t_{n}=1\right\}$ of $[0,1]$. Clearly

$$
\lim _{|\pi| \downarrow 0} \sup _{0 \leqslant t \leqslant 1}\left|X_{t}-X_{t}^{\pi}\right|=0
$$

for all $\omega \in \Omega$. The process $X_{t}^{\pi}$ can be decomposed as follows:

$$
\begin{aligned}
X_{t}^{\pi}= & X_{0}+\int_{0}^{t} \sigma\left(X_{s}^{\pi}\right) \dot{M}_{s}^{\pi} \mathrm{d} s+\int_{0}^{t} b\left(h\left(Y_{s}, M_{s}\right)\right) \\
& \times \exp \left(\int_{M_{s}}^{M_{s}^{\pi}} \sigma^{\prime}\left(h\left(Y_{s}, z\right)\right) \mathrm{d} z\right) \mathrm{d} A_{s}:=A_{t}^{1}+A_{t}^{2},
\end{aligned}
$$

where

$$
\begin{aligned}
A_{t}^{1} & =\int_{0}^{t} \sigma\left(X_{s}^{\pi}\right) \dot{M}_{s}^{\pi} \mathrm{d} s \\
& =\int_{0}^{t}\left\{\sigma\left(X_{s}^{\pi}\right)-\sigma\left(X_{s}\right)\right\} \dot{M}_{s}^{\pi} \mathrm{d} s+\int_{0}^{t} \sigma\left(X_{s}\right) \dot{M}_{s}^{\pi} \mathrm{d} s:=B_{t}^{1}+B_{t}^{2} .
\end{aligned}
$$

Using the fact that $\sup _{0 \leqslant s \leqslant 1}\left|M_{s}^{\pi}-M_{s}\right|$ converges to zero as $|\pi|$ tends to zero, we easily deduce that

$$
\lim _{|\pi| \downarrow 0} \sup _{0 \leqslant t \leqslant 1}\left|A_{t}^{2}-\left(X_{0}+\int_{0}^{t} b\left(X_{s}\right) \mathrm{d} A_{s}\right)\right|=0,
$$

for all $\omega \in \Omega$. As a consequence,

$$
\lim _{|\pi| \downarrow 0} \sup _{0 \leqslant t \leqslant 1}\left|A_{t}^{1}-\left(X_{t}-X_{0}-\int_{0}^{t} b\left(X_{s}\right) \mathrm{d} A_{s}\right)\right|=0,
$$

for all $\omega \in \Omega$. Hence, taking into account Definition 2.1, in order to show that the process $X_{t}$ satisfies (2.1) it only remains to prove that $B_{t}^{1}$ converges to zero in probability, uniformly in $t$, as $|\pi|$ tends to zero. We can write

$$
\begin{aligned}
\sigma\left(X_{s}^{\pi}\right)-\sigma\left(X_{s}\right) & =\sigma\left(h\left(Y_{s}, M_{s}^{\pi}\right)\right)-\sigma\left(h\left(Y_{s}, M_{s}\right)\right) \\
& =\sigma^{\prime} \sigma\left(X_{s}\right)\left(M_{s}^{\pi}-M_{s}\right)+\frac{1}{2}\left\{\sigma\left(\sigma^{\prime}\right)^{2}+\sigma^{2} \sigma^{\prime \prime}\right\}\left(h\left(Y_{s}, \xi\right)\right)\left(M_{s}^{\pi}-M_{s}\right)^{2},
\end{aligned}
$$


where $\xi$ belongs to the interval defined by $M_{s}^{\pi}$ and $M_{s}$. We have

$$
\begin{gathered}
\left|\int_{0}^{t}\left\{\sigma\left(\sigma^{\prime}\right)^{2}+\sigma^{2} \sigma^{\prime \prime}\right\}\left(h\left(Y_{s}, \xi\right)\right)\left(M_{s}^{\pi}-M_{s}\right)^{2} \dot{M}_{s}^{\pi} \mathrm{d} s\right| \leqslant C(\omega) \int_{0}^{t}\left|\dot{M}_{s}^{\pi}\right|\left(M_{s}^{\pi}-M_{s}\right)^{2} \mathrm{~d} s \\
\leqslant C(\omega) \sup _{|u-v| \leqslant|\pi|}\left|M_{u}-M_{v}\right| \int_{0}^{t}\left|\dot{M}_{s}^{\pi}\right|\left|M_{s}^{\pi}-M_{s}\right| \mathrm{d} s .
\end{gathered}
$$

The last factor in the above expression can be estimated as follows:

$$
\begin{aligned}
\int_{0}^{1}\left|\dot{M}_{s}^{\pi}\right|\left|M_{s}^{\pi}-M_{\mathrm{s}}\right| \mathrm{d} s & \leqslant \sum_{i=0}^{n-1} \frac{\left|\Delta_{i} M\right|}{\Delta_{i} t} \int_{t_{i}}^{t_{i+1}}\left(\frac{\left|\Delta_{i} M\right|}{\Delta_{i} t}\left(s-t_{i}\right)+\left|M_{s}-M_{t_{i}}\right|\right) \mathrm{d} s \\
& \leqslant \frac{1}{2} \sum_{i=0}^{n-1}\left|\Delta_{i} M\right|^{2}+\sum_{i=0}^{n-1} \sup _{s \in\left[t_{i}, t_{i+1}\right]}\left(M_{s}-M_{t_{i}}\right)^{2},
\end{aligned}
$$

where $\Delta_{i} t=t_{i+1}-t_{i}$ and $\Delta_{i} M=M_{t_{i+1}}-M_{t_{i}}$. As a consequence we deduce that

$$
\lim _{K \uparrow \infty} \sup _{\pi} P\left(\int_{0}^{1}\left|\dot{M}_{s}^{\pi}\right|\left|M_{s}^{\pi}-M_{s}\right| \mathrm{d} s>K\right)=0 .
$$

Hence (2.2) converges to zero in probability, uniformly in $t$, as $|\pi|$ tends to zero. Finally, the convergence to zero of the term $\int_{0}^{t} \sigma^{\prime} \sigma\left(X_{s}\right)\left(M_{s}^{\pi}-M_{s}\right) \dot{M}_{s}^{\pi} \mathrm{d} s$ follows from Lemma 2.3 below.

Lemma 2.3. Let $\Phi=\left\{\Phi_{t}, t \in[0,1]\right\}$ be a continuous stochastic process. Then

$$
\lim _{|\pi| \downarrow 0} P\left(\sup _{0 \leqslant t \leqslant 1}\left|\int_{0}^{t} \Phi_{s}\left(M_{s}^{\pi}-M_{s}\right) \dot{M}_{s}^{\pi} \mathrm{d} s\right|>\epsilon\right)=0,
$$

for all $\epsilon>0$.

Proof. Fix a partition $\pi=\left\{0=t_{0}<t_{1}<\ldots<t_{n}=1\right\}$ and consider the partition given by $s_{j}=j / m, 0 \leqslant j \leqslant m$. We can make the following estimation:

$$
\begin{aligned}
\left|\int_{0}^{t} \Phi_{s}\left(M_{s}^{\pi}-M_{s}\right) \dot{M}_{s}^{\pi} \mathrm{d} s\right| \leqslant & \sum_{j=0}^{m-1}\left|\Phi_{s_{j}} \int_{s_{j} \wedge t}^{s_{j+1} \wedge t}\left(M_{s}^{\pi}-M_{s}\right) \dot{M}_{s}^{\pi} \mathrm{d} s\right| \\
& +\left|\sum_{j=0}^{m-1} \int_{s_{j} \wedge t}^{s_{j+1} \wedge t}\left(\Phi_{s}-\Phi_{s_{j}}\right)\left(M_{s}^{\pi}-M_{s}\right) \dot{M}_{s}^{\pi} \mathrm{d} s\right|:=C_{1}(t)+C_{2}(t) .
\end{aligned}
$$

We have that $\lim _{|\pi| \downarrow 0} P\left(\sup _{0 \leqslant t \leqslant 1}\left|C_{1}(t)\right|>\epsilon\right)=0$ for all $\epsilon>0$. In fact,

$$
\int_{s_{j} \wedge t}^{s_{j+1} \wedge t}\left(M_{s}^{\pi}-M_{s}\right) \dot{M}_{s}^{\pi} \mathrm{d} s=\frac{1}{2}\left\{\left(M_{s_{j+1} \wedge t}^{\pi}\right)^{2}-\left(M_{s_{j} \wedge t}^{\pi}\right)^{2}\right\}-\int_{s_{j} \wedge t}^{s_{j+1} \wedge t} M_{s} \dot{M}_{s}^{\pi} \mathrm{d} s .
$$

Then, applying a result of Yor (1977), and using the absolute continuity of the quadratic 
variation of $\langle M\rangle$, we deduce that $\int_{s_{j} \wedge t}^{s_{j+1} \wedge t} M_{s} \dot{M}_{s}^{\pi} \mathrm{d} s$ converges in probability, uniformly in $t$ as $|\pi|$ tends to zero, to

$$
\frac{1}{2}\left\{\left(M_{s_{j+1} \wedge t}^{\pi}\right)^{2}-\left(M_{s_{j} \wedge t}^{\pi}\right)^{2}\right\} .
$$

For the term $C_{2}(t)$ we have

$$
\left|C_{2}(t)\right| \leqslant \sup _{|s-t| \leqslant 1 / m}\left|\Phi_{s}-\Phi_{t}\right| \int_{0}^{1}\left|M_{s}^{\pi}-M_{\mathrm{s}}\right|\left|\dot{M}_{s}^{\pi}\right| \mathrm{d} s,
$$

and this converges to zero in probability, uniformly in $t$, as $m$ tends to infinity, namely,

$$
\lim _{m \rightarrow \infty} \lim _{|\pi| \downarrow 0} P\left(\sup _{0 \leqslant t \leqslant 1}\left|C_{2}(t)\right|>\epsilon\right)=0,
$$

for all $\epsilon>0$, taking into account the estimate (2.3).

Using the same method of proof as in Theorem 2.2 one can deduce an Itô formula for $f\left(X_{t}\right)$, for $f \in C^{2}$. This remark is also valid for the equations to be studied in the subsequent sections.

Now we shall state the conditions for uniqueness of solutions to (2.1). Let $A$ be the set of continuous processes $X$ such that there exists an approximation sequence of processes $M^{n}$ with absolutely continuous trajectories, verifying the following properties.

(a) $M_{t}^{n} \rightarrow M_{t}$ for all $t$, a.s.

(b) The unique solution $X^{n}$ of the equation

$$
X_{t}^{n}=X_{0}+\int_{0}^{t} b\left(X_{s}^{n}\right) \mathrm{d} A_{s}+\int_{0}^{t} \sigma\left(X_{s}^{n}\right) \dot{M}_{s}^{n} \mathrm{~d} s,
$$

converges to $X_{t}$ for each $t$, a.s.

Theorem 2.4. Under the assumptions of Theorem 2.2, the solution to (2.1) is unique in the class A.

Proof. It is not difficult to prove that $X_{t}^{n}=h\left(Y_{t}^{n}, M_{t}^{n}\right)$ where $Y_{t}^{n}$ satisfies

$$
Y_{t}^{n}=X_{0}+\int_{0}^{t} f\left(Y_{s}^{n}, M_{s}^{n}\right) \mathrm{d} A_{s} .
$$

Using the particular expression for the function $f$ and the convergences of $X^{n}$ and $M^{n}$ we can show that there exists a random variable $K_{1}$ such that

$$
\left|f\left(Y_{s}, M_{s}^{n}\right)-f\left(Y_{s}^{n}, M_{s}^{n}\right)\right| \leqslant K_{1}\left|Y_{s}-Y_{s}^{n}\right|, \quad \text { a.s. }
$$

Then from Gronwall's lemma it follows that $Y_{t}^{n} \rightarrow Y_{t}$ for all $t$, a.s. Therefore, by definition of A one has that $X=h(Y, M)$ and therefore the uniqueness follows.

In the multidimensional case, similar results can be obtained under the Frobenius condition. 


\section{An anticipating semilinear equation}

In this section we study a semilinear equation that is different from the case studied in the previous section. The equation we study here is

$$
X_{t}=X_{0}+\int_{0}^{t} b\left(s, X_{s}\right) \mathrm{d} A_{s}+\int_{0}^{t} \sigma_{s} X_{s} \circ \mathrm{d} M_{s},
$$

where $M$ is a continuous local martingale such that $M_{0}=0$ and $\langle M\rangle_{t}=\int_{0}^{t} m_{s} \mathrm{~d} s$, and $A$ is a continuous bounded variated process. The coefficients $b: \Omega \times[0,1] \times \mathbb{R} \rightarrow \mathbb{R}$ and $\sigma: \Omega \times[0,1] \rightarrow \mathbb{R}$ are measurable functions such that the following hold.

(H1) For all $\omega \in \Omega, s \in[0,1]$ and $x, y \in \mathbb{R}$ we have

$$
\begin{gathered}
|b(\omega, s, x)-b(\omega, s, y)| \leqslant K(\omega)|x-y|, \\
|b(\omega, s, 0)| \leqslant K(\omega),
\end{gathered}
$$

for some random variable $K$.

(H2) $\sigma$ is Stratonovich integrable and the family of random variables

$$
\xi_{\pi}:=\int_{0}^{1}\left|\int_{0}^{r} \sigma_{s} \dot{M}_{s}^{\pi} \mathrm{d} s-\int_{0}^{r} \sigma_{s} \circ \mathrm{d} M_{s}\right|\left|\sigma_{r} \dot{M}_{r}^{\pi}\right| \mathrm{d} r,
$$

where $\pi$ runs over the set of all partitions of $[0,1]$, is bounded in probability, namely, $\lim _{K \uparrow \infty} \sup _{\pi} P\left(\left|\xi_{\pi}\right|>K\right)=0$.

(H3)

$$
\lim _{|\pi| \downarrow 0} P\left(\sup _{0 \leqslant t \leqslant 1}\left|\int_{0}^{t}\left(\int_{0}^{r} \sigma_{s} \dot{M}_{s}^{\pi} \mathrm{d} s-\int_{0}^{r} \sigma_{s} \circ \mathrm{d} M_{s}\right) \sigma_{r} \dot{M}_{r}^{\pi} \mathrm{d} r\right|>\epsilon\right)=0,
$$

for all $\epsilon>0$.

It is not difficult to see that, in the case when $\sigma$ is absolutely continuous and $\int_{0}^{1}\left|\dot{\sigma}_{s}\right| \mathrm{d} s<\infty$, then the conditions (H2) and (H3) are satisfied. Now we define the class where existence and uniqueness will be given. Let $A$ be the class of continuous processes $X$ such that the following is true.

(H4) $\sigma_{t} X_{t}$ is Stratonovich integrable, and

$$
\lim _{|\pi| \downarrow 0} \lim _{\left|\pi^{\prime}\right| \downarrow 0} P\left(\left|\int_{0}^{t} \sigma_{s} X_{s} \exp \left(-\int_{0}^{s} \sigma_{r} \dot{M}_{r}^{\pi} \mathrm{d} r\right)\left(\dot{M}_{s}^{\pi^{\prime}}-\dot{M}_{s}^{\pi}\right) \mathrm{d} s\right|>\epsilon\right)=0,
$$

for any $t \in[0,1]$ and $\epsilon>0$.

Theorem 3.1. Assume conditions $(\mathrm{H} 1)-(\mathrm{H} 3)$. Then, there is a unique solution to (3.1) in the class A. Furthermore this solution is given by

$$
X_{t}=\exp \left(\int_{0}^{t} \sigma_{s} \circ \mathrm{d} M_{s}\right) X_{0}+\int_{0}^{t} \exp \left(\int_{u}^{t} \sigma_{s} \circ \mathrm{d} M_{s}\right) b\left(u, X_{u}\right) \mathrm{d} A_{u} .
$$


Proof. First, it is clear that $X$ in (3.3) is well defined. In order to show that $X$ belongs to the class $A$ and satisfies (3.1) we first claim that for any continuous process $\Phi=$ $\left\{\Phi_{s}, s \in[0,1]\right\}$ we have

$$
\lim _{|\pi| \downarrow 0} P\left(\sup _{0 \leqslant t \leqslant 1}\left|\int_{0}^{t} \Phi_{s} \sigma_{s}\left\{\exp \left(\int_{0}^{s} \sigma_{r} \dot{M}_{r}^{\pi} \mathrm{d} r\right)-\exp \left(\int_{0}^{s} \sigma_{r} \circ \mathrm{d} M_{r}\right)\right\} \dot{M}_{s}^{\pi} \mathrm{d} s\right|>\epsilon\right)=0,
$$

for any $\epsilon>0$. This follows by using a similar argument as in the proofs of Theorem 2.2 and Lemma 2.3, using assumptions $(\mathrm{H} 2)$ and $(\mathrm{H} 3)$ and developing the exponential up to the second order.

Let us now show the Stratonovich integrability of $\sigma X$. Consider the approximation $\int_{0}^{t} \sigma_{s} X_{s} \dot{M}_{s}^{\pi} \mathrm{d} s=\int_{0}^{t} \sigma_{r} \exp \left(\int_{0}^{s} \sigma_{r} \circ \mathrm{d} M_{r}\right)\left\{X_{0}+\int_{0}^{s} \exp \left(-\int_{0}^{u} \sigma_{r} \circ \mathrm{d} M_{r}\right) b\left(u, X_{u}\right) \mathrm{d} A_{u}\right\} \dot{M}_{s}^{\pi} \mathrm{d} s$.

From (3.4) with

$$
\Phi_{t}=X_{0}+\int_{0}^{s} \exp \left(-\int_{0}^{u} \sigma_{r} \circ \mathrm{d} M_{r}\right) b\left(u, X_{u}\right) \mathrm{d} A_{u}
$$

we get that

$$
\sup _{0 \leqslant t \leqslant 1}\left|\int_{0}^{t} \sigma_{s} X_{s} \dot{M}_{s}^{\pi} \mathrm{d} s-B_{t}^{\pi}\right|
$$

converges in probability to zero as $|\pi|$ tends to zero, where

$$
B_{t}^{\pi}=\int_{0}^{t} \sigma_{s} \exp \left(\int_{0}^{s} \sigma_{r} \dot{M}_{r}^{\pi} \mathrm{d} r\right)\left\{X_{0}+\int_{0}^{s} \exp \left(-\int_{0}^{u} \sigma_{r} \circ \mathrm{d} M_{r}\right) b\left(u, X_{u}\right) \mathrm{d} A_{u}\right\} \dot{M}_{s}^{\pi} \mathrm{d} s .
$$

Next one uses integration by parts to deduce that $\int_{0}^{t} \sigma_{s} X_{s} \dot{M}_{s}^{\pi} \mathrm{d} s$ converges in probability, uniformly with respect to $t$ to

$$
X_{t}-X_{0}-\int_{0}^{t} b\left(u, X_{u}\right) \mathrm{d} A_{u}
$$

This proof also shows that $X$ satisfies (3.1).

Assuming that $X$ satisfies (3.1), and $\sigma X$ is Stratonovich integrable, let us show that (3.2) holds. One can write, using integration by parts as well as (3.1),

$$
\begin{aligned}
\lim _{\left|\pi^{\prime}\right| \downarrow 0} & \int_{0}^{t} \sigma_{s} X_{s} \exp \left(-\int_{0}^{s} \sigma_{r} \dot{M}_{r}^{\pi} \mathrm{d} r\right) \dot{M}_{s}^{\pi^{\prime}} \mathrm{d} s \\
= & \int_{0}^{t} \sigma_{s} X_{s} \exp \left(-\int_{0}^{s} \sigma_{r} \dot{M}_{r}^{\pi} \mathrm{d} r\right) M_{s}^{\pi} \mathrm{d} s-X_{0}+X_{t} \exp \left(-\int_{0}^{t} \sigma_{s} \dot{M}_{s}^{\pi} \mathrm{d} s\right) \\
& -\int_{0}^{t} \exp \left(-\int_{0}^{s} \sigma_{r} \dot{M}_{r}^{\pi} \mathrm{d} r\right) b\left(s, X_{s}\right) \mathrm{d} A_{s},
\end{aligned}
$$


in the sense of the convergence in probability. Taking the limit as $|\pi|$ tends to zero, and using (3.3), one obtains (3.2).

Now we proceed with the proof of the uniqueness. For this, let $X \in \mathrm{A}$ such that it satisfies (3.1). As before the limit (3.5) holds. Finally, taking the limit as $|\pi|$ tends to zero in both members of (3.5) and using (3.2) we deduce that $X$ satisfies (3.3).

\section{Multidimensional Stratonovich equations}

In this section we shall present two cases of multidimensional Stratonovich equations where we can use the technique introduced in Section 2 in order to establish the existence and uniqueness of a solution. We shall assume that $W=\left\{W_{t}, t \in[0,1]\right\}$ is an $m$-dimensional Wiener process defined on a filtered probability space $\left(\Omega, \mathrm{F}, P,\left\{\mathrm{~F}{ }_{t}, t \in[0,1]\right\}\right)$.

\subsection{STRATONOVICH EQUATIONS WITH RANDOM DRIFT AND INITIAL CONDITION}

We shall deal with the case of a multidimensional Stratonovich stochastic differential equation driven by $W$, where $\sigma$ is a deterministic function, the coefficients $b$ and $\sigma$ depend on the time variable, and $b$ and $X_{0}$ may be random. Consider the stochastic differential equation in $\mathbb{R}^{d}$ :

$$
X_{t}=X_{0}+\int_{0}^{t} b\left(s, X_{s}\right) \mathrm{d} s+\sum_{i=1}^{m} \int_{0}^{t} \sigma^{i}\left(s, X_{s}\right) \circ \mathrm{d} W_{s}^{i},
$$

where $X_{0} \in L^{0}\left(\Omega ; \mathbb{R}^{d}\right)$, and $b: \Omega \times[0,1] \times \mathbb{R}^{d} \rightarrow \mathbb{R}^{d}$ and $\sigma^{i}:[0,1] \times \mathbb{R}^{d} \rightarrow \mathbb{R}^{d}, 1 \leqslant$ $i \leqslant m$ are measurable functions. Ocone and Pardoux (1989) proved by means of a generalized version of the Itô-Ventzell formula that (4.1) possesses a unique non-exploding solution provided that $X_{0}$ and $b$ are smooth in the sense of Malliavin calculus. By a solution of (4.1) we mean a $d$-dimensional continuous process $X=\left\{X_{t}, t \in[0,1]\right\}$ such that $\sigma_{i}\left(s, X_{s}\right)$ is Stratonovich integrable with respect to $W^{i}$ in the sense of Definition 2.1, properly extended to multidimensional processes, and (4.1) holds a.s. for all $t \in[0,1]$. Define

$$
m(t, x)=\frac{1}{2} \sum_{i=1}^{m}\left(\frac{\partial \sigma^{i}}{\partial x} \sigma^{i}\right)(t, x) .
$$

Theorem 4.1. Assume that $\sigma^{i}(t, \cdot) \in C^{3}\left(\mathbb{R}^{d}, \mathbb{R}^{d}\right)$ for $t \in[0,1], 1 \leqslant i \leqslant m, \sigma^{\mathrm{i}}(t, \cdot)$ has bounded partial derivatives of first order and $\sigma^{i}(t, 0)$ is bounded. Also suppose that $m(t, x)$ is Lipschitz in $x$ uniformly with respect to $t, b(\omega, t, x)$ is $C^{1}$ in $x, \sigma(t, x)$ is of class $C^{1}$ in $t$, and

(i) for all $\epsilon>0,|b(\omega, t, x)| \leqslant C_{\epsilon}\left(1+|x|^{1-\epsilon}\right)$, for all $(\omega, t, x) \in \Omega \times[0,1] \times \mathbb{R}^{d}$;

(ii) $|(\partial b / \partial x)(\omega, t, x)| \leqslant C_{K}(\omega)$, for all $(\omega, t, x) \in \Omega \times[0,1] \times \mathbb{R}^{d}$ with $|x| \leqslant K$.

Then there exists a solution to (4.1). 
Proof. Let $\varphi_{t}(x)$ denote the flow defined by the adapted equation

$$
\begin{aligned}
\varphi_{t}(x) & =x+\int_{0}^{t} \sigma^{i}\left(s, \varphi_{s}(x)\right) \circ \mathrm{d} W_{s}^{i} \\
& =x+\int_{0}^{t} \sigma^{i}\left(s, \varphi_{s}(x)\right) \mathrm{d} W_{s}^{i}+\int_{0}^{t} m\left(s, \varphi_{s}(x)\right) \mathrm{d} s,
\end{aligned}
$$

where we make the convention of summation over repeated indices. Under the hypothesis of the theorem, $\varphi_{t}$ is a $C^{1}$ diffeomorphism of $\mathbb{R}^{d}$ and $\left(\partial \varphi_{t} / \partial x\right)^{-1}$ exists. Let $Y=\left\{Y_{t}, t \in[0,1]\right\}$ be the unique solution of

$$
\begin{aligned}
\frac{\mathrm{d} Y_{t}}{\mathrm{~d} t} & =\left(\frac{\partial \varphi_{t}}{\partial x}\right)^{-1}\left(Y_{t}\right) b\left(t, \varphi_{t}\left(Y_{t}\right)\right), \\
Y_{0} & =X_{0} .
\end{aligned}
$$

Existence and uniqueness of a solution to (4.2) follows from the fact that $\left(\partial \varphi_{t} / \partial x\right)^{-1}(x) b\left(\omega, t, \varphi_{t}(x)\right)$ is $C^{1}$ in $x$ and a.s. locally bounded together with its derivative in $x$. The fact that the solution is global follows from the estimate

$$
\left|\left(\frac{\partial \varphi_{t}}{\partial x}\right)^{-1}(x) b\left(\omega, t, \varphi_{t}(x)\right)\right| \leqslant K_{\epsilon}(\omega)\left(1+|x|^{1-\epsilon}\right) \text {, for all } t,
$$

which can be deduced from our hypothesis as in the case of Ocone and Pardoux (1989, p. 64).

Now we shall show the existence of a solution to (4.1). Define $X_{t}=\varphi_{t}\left(Y_{t}\right)$ and $X_{t}^{\pi}=\varphi_{t}^{\pi}\left(Y_{t}\right)$ where $\pi=\left\{0=t_{0}<t_{1}<\ldots<t_{n}=1\right\}$ is a partition of $[0,1]$, and

$$
\varphi_{t}^{\pi}(x)=x+\int_{0}^{t} \sigma^{i}\left(s, \varphi_{s}(x)\right)\left(\dot{W}_{s}^{i}\right)^{\pi} \mathrm{d} s .
$$

We have

$$
\mathrm{d} X_{t}^{\pi}=\frac{\partial \varphi_{t}^{\pi}}{\partial x}\left(Y_{t}\right)\left(\frac{\partial \varphi_{t}}{\partial x}\right)^{-1}\left(Y_{t}\right) b\left(t, \varphi_{t}\left(Y_{t}\right)\right) \mathrm{d} t+\sigma^{i}\left(t, \varphi_{t}\left(Y_{t}\right)\right)\left(\dot{W}_{t}^{i}\right)^{\pi} \mathrm{d} t .
$$

As $\left(\varphi_{t}^{\pi}(x),\left(\partial \varphi_{t}^{\pi} / \partial x\right)(x)\right)$ converges to $\left(\varphi_{t}(x),\left(\partial \varphi_{t} / \partial x\right)(x)\right)$ in probability uniformly for $(t, x) \in[0,1] \times[-K, K]^{d}$, for all $K>0$, when $|\pi|$ tends to zero, we then have that

$$
X_{t}^{\pi} \rightarrow \varphi_{t}\left(Y_{t}\right)
$$

and

$$
\frac{\partial \varphi_{t}^{\pi}}{\partial x}\left(Y_{t}\right)\left(\frac{\partial \varphi_{t}}{\partial x}\right)^{-1}\left(Y_{t}\right) \rightarrow I
$$

in probability, uniformly in $t \in[0,1]$. Hence

$$
\int_{0}^{t} \sigma^{i}\left(s, \varphi_{s}(x)\right)\left(\dot{W}_{s}^{i}\right)^{\pi} \mathrm{d} s \rightarrow X_{t}-X_{0}-\int_{0}^{t} b\left(s, \varphi_{s}\left(Y_{s}\right)\right) \mathrm{d} s,
$$

in probability, uniformly for $t \in[0,1]$. 
The uniqueness is done in a similar way as in the one-dimensional case. For this let $A$ be the class of continuous processes $X$ such that there exists an approximation sequence of processes $W^{n}$ with absolutely continuous paths such that the following hold.

(a) $W_{t}^{n} \rightarrow W_{t}$ for all $t$, a.s.

(b) The unique solution $X^{n}$ of the equation

$$
X_{t}^{n}=X_{0}+\int_{0}^{t} b\left(s, X_{s}^{n}\right) \mathrm{d} s+\int_{0}^{t} \sigma^{i}\left(s, X_{s}^{n}\right) \dot{W}_{s}^{i, n} \mathrm{~d} s,
$$

converges to $X_{t}$ for each $t$, a.s.

(c) $\left(\varphi_{t}^{n}(x),\left(\partial \varphi_{t}^{n} / \partial x\right)^{-1}(x)\right)$ converges to $\left(\varphi_{t}(x),\left(\partial \varphi_{t} / \partial x\right)^{-1}(x)\right)$ uniformly in $t$ and for $x$ in compacts. Here $\varphi_{t}^{n}$ is defined through (4.3) using $W^{n}$ instead of $W^{\pi}$.

Note that for any refining sequence $\left\{\pi_{n}\right\}$ of partitions of $[0,1]$ such that $\left|\pi_{n}\right| \downarrow 0, W^{\pi_{n}}$ satisfies properties (a) and (c) above if $\sigma$ and $b$ are three times differentiable with Lipschitz derivatives as can be seen in Kunita (1990, Section 5.7).

Theorem 4.2. Under the assumptions of Theorem 4.1, there is a unique solution to (4.1) in the class $\mathrm{A}$.

Proof. The proof in this case is similar to the one-dimensional case. It is not difficult to see that $X^{n}=\varphi^{n}\left(Y^{n}\right)$ where $Y^{n}$ solves (4.2) with $\varphi$ replaced by $\varphi^{n}$. Using properties (b) and (c) above, it follows that the solution has to be unique.

\subsection{STOCHASTIC DIFFERENTIAL EQUATIONS WITH BOUNDARY CONDITIONS}

We shall use our techniques to prove existence and uniqueness for stochastic differential equations with boundary conditions of the type

$$
\begin{aligned}
& X_{t}=X_{0}+\int_{0}^{t} b\left(X_{s}\right) \mathrm{d} s+\int_{0}^{t} \sigma\left(X_{s}\right) \circ \mathrm{d} W_{s}, \\
& V_{0}=H_{0} X_{0}+H_{1} X_{1},
\end{aligned}
$$

where $H_{0}$ and $H_{1}$ are $\mathrm{d} \times \mathrm{d}$ matrices. This type of equation has been recently studied by Garnier (1995) using a stochastic embedding method. To introduce the hypotheses under which we prove existence, we need to define $\varphi_{t}(x)$ as the flow associated with (4.4). Also define $Z(t, x)=H_{1}(\partial / \partial x) \varphi_{t}\left(\left(H_{0}+H_{1}\right)^{-1} x\right)+H_{0}\left(H_{0}+H_{1}\right)^{-1}$. Subsequently we shall assume the following.

(H ) The functions $b, \sigma: \mathbb{R}^{d} \rightarrow \mathbb{R}^{d}$ are three times differentiable with uniformly bounded and continuous partial derivatives. Furthermore assume that there exists a positive constant $\alpha$ such that $|\operatorname{det} Z(t, x)| \geqslant \alpha$ for all $(t, x) \in[0,1] \times \mathbb{R}^{d}$, almost surely. 
Theorem 4.3. Assume condition (H ). Then there exists a solution to (4.4) given by $\varphi_{t}\left(\left(H_{0}+H_{1}\right)^{-1} \bar{V}(1)\right)$, where $\bar{V}$ is the unique solution of the following adapted stochastic differential equation:

$$
\begin{aligned}
\bar{V}_{t}= & V_{0}-\int_{0}^{t}\left(Z^{-1} H_{1} b\left(\varphi\left(\left(H_{0}+H_{1}\right)^{-1} \cdot\right)\right)\right)\left(s, \bar{V}_{s}\right) \mathrm{d} s \\
& -\int_{0}^{t}\left(Z^{-1} H_{1} \sigma\left(\varphi\left(\left(H_{0}+H_{1}\right)^{-1} \cdot\right)\right)\right)\left(s, \bar{V}_{s}\right) \circ \mathrm{d} W_{s} .
\end{aligned}
$$

Proof. First we want to check that the boundary condition is satisfied. For this it is enough to see Lemma 1 in Garnier (1995). Now we prove that (4.4) holds. For this we shall need the following evaluation formula:

$$
\left.\int_{0}^{t} \sigma\left(\varphi_{s}(x)\right) \circ \mathrm{d} W_{s}\right|_{x=X_{0}}=\int_{0}^{t} \sigma\left(\varphi_{s}\left(X_{0}\right)\right) \circ \mathrm{d} W_{s},
$$

where $X_{0}$ is a random variable that belongs to $L^{p}(\Omega)$ for some $p>1$. This formula is proven, for instance, in Theorem 5.3.3 and Theorem 6.1.1 of Nualart (1997).

Therefore to finish the proof we only need to prove that $\bar{V}(1) \in L^{p}(\Omega)$ for some $p>1$. This is obtained using standard $L^{p}$ estimation methods in (4.5). In particular we need to use the fact that $\left|\operatorname{det} Z^{-1}(t, x)\right| \leqslant \alpha^{-1}$ uniformly for $(t, x)$. From here, the existence follows.

For uniqueness we define as before a class $A$ of continuous processes such that there exists an approximation with almost surely absolutely continuous paths $W^{n}$ such that the following hold.

(a) $W_{t}^{n} \rightarrow W_{t}$ for all $t$, a.s.

(b) The unique solution $X^{n}$ of the equation

$$
\begin{aligned}
X_{t}^{n} & =X_{0}^{n}+\int_{0}^{t} b\left(X_{s}^{n}\right) \mathrm{d} s+\int_{0}^{t} \sigma\left(X_{s}^{n}\right) \dot{W}_{s}^{n} \mathrm{~d} s, \\
V_{0} & =H_{0} X_{0}^{n}+H_{1} X_{1}^{n}
\end{aligned}
$$

converges to $X_{t}$ for each $t$, a.s.

(c) $\left.\varphi_{t}^{n}(x),\left(\partial \varphi_{t}^{n} / \partial x\right)(x)\right)$ converges to $\left(\varphi_{t}(x),\left(\partial \varphi_{t} / \partial x\right)(x)\right)$ uniformly in $t$ and for $x$ in compacts. Here $\varphi_{t}^{n}$ is a flow that is defined through (4.4) using $W^{n}$ instead of $W$.

In assumption (b) one is supposing that the equation has a unique solution. Conditions for (c) to be satisfied have been thoroughly studied in recent years (see, for example, Ikeda and Watanabe 1981).

As in the case of the Ocone-Pardoux equation, it is not difficult to see that $W^{\pi_{n}}$ satisfies the above conditions (a) and (c), for any refining sequence $\left\{\pi_{n}\right\}$ of partitions of $[0,1]$ such that $\left|\pi_{n}\right| \downarrow 0$, considering the results obtained by Gyöngy (1987). 
Theorem 4.4. Assume condition (H ). Then there is a unique solution to (4.4) in the class A.

Proof. The proof here is similar to the previous uniqueness proofs. Here we have that by (c) it is enough to prove that $X_{0}^{n} \rightarrow X_{0}$ a.s. To obtain this result it is enough to apply Lemma 6, Section 5.2, in Garnier (1995).

\section{Acknowledgements}

This work was supported by Dirección General de Investigación Científica y Técnica Grant PB93-0052 (to David Nualart) Universitat Pompeu Fabra-Fundació Universitat Nova Grant PS95-005 (to Arturs Kohatsu-Higa) and a Consejo Nacional de Ciencia y Tecnologia de Mexico Grant (to Jorge A. León).

\section{References}

Doss, H. (1977) Liens entre équations différentielles stochastiques et ordinaires. Ann. Inst. Henri Poincaré, Sect. B, 13, 99-125.

Garnier, J. (1995) Stochastic invariant imbedding. Application to stochastic differential equations with boundary conditions. Probab. Theory Related Fields, 102, 249-271.

Gyöngy, I. (1987) on the approximation of stochastic differential equations. Stoch. Stoch. Rep., 23, $331-352$.

Ikeda, N. and Watanabe, S. (1981) Stochastic Differential Equations and Diffusion Processes. Amsterdam: North-Holland.

Kohatsu-Higa, A. and León, J. (1997) Anticipating stochastic differential equations of Stratonovich type. Appl. Math. Opt. To appear.

Kunita, H. (1990) Stochastic Flows and Stochastic Differential Equations. Cambridge Studies in Advanced Mathematics 21. Cambridge: Cambridge University Press.

Millet, A., Nualart, D. and Sanz, M. (1992) Large deviations for a class of anticipating stochastic differential equations. Ann. Probab., 20, 1902-1931.

Nualart, D. (1997) Analysis on the Wiener and anticipating stochastic calculus. École d'Été de Probabilités de Saint Flour 1995. Lecture Notes in Math. Berlin: Springer-Verlag. To appear.

Nualart, D. and Pardoux, E. (1988) Stochastic calculus with anticipating integrands. Probab. Theory Related Fields, 78, 535-581.

Ocone, D. and E. Pardoux, (1989) A generalized Itô-Ventzell formula. Application to a class of anticipating stochastic differential equations. Ann. Inst. Henri Poincaré, 25, 39-71.

Russo, F. and Vallois, P. (1993) Forward, backward and symmetric stochastic integration. Probab. Theory Related Fields, 97, 403-421.

Yor, M. (1997) Sur quelques approximations d'intégrales stochastiques. Seminaire de Probabilités XI, pp. 518-528. Lecture Notes in Math. Berlin: Springer-Verlag.

Received May 1996 and revised November 1996 